\title{
Tuberculosis Transmission by Patients with Smear- Negative Pulmonary Tuberculosis in a Large Cohort in The Netherlands
}

\author{
Alma Tostmann, ${ }^{1,2}$ Sandra V. Kik, ${ }^{3,4}$ Nico A. Kalisvaart, ${ }^{3}$ Maruschka M. Sebek, ${ }^{3}$ Suzanne Verver, ${ }^{3,4}$ Martin J. Boeree, ${ }^{1,2}$ \\ and Dick van Soolingen ${ }^{5}$ \\ 'Department of Pulmonary Diseases, Radboud University Nijmegen Medical Centre, Nijmegen, ${ }^{2}$ University Lung Centre Dekkerswald, Groesbeek, \\ ${ }^{3}$ Royal Netherlands Tuberculosis Association, The Hague, ${ }^{4}$ Center for Infection and Immunity Amsterdam, Academic Medical Centre, Amsterdam, \\ and ${ }^{5}$ National Institute for Public Health and the Environment, Bilthoven, The Netherlands
}

Background. Sputum smear microscopy is commonly used for diagnosing tuberculosis (TB). Although patients with sputum smear-negative TB are less infectious than patients with smear-positive TB, they also contribute to TB transmission. The objective of this study was to determine the proportion of TB transmission events caused by patients with smear-negative pulmonary TB in The Netherlands.

Methods. All patients in The Netherlands with culture-confirmed TB during the period 1996-2004 were included in this study. Patients with identical DNA fingerprints in Mycobacterium tuberculosis isolates from sputum samples were clustered. The first patients in a cluster were considered to be the index patients; all other patients were considered to have secondary cases. In addition, we examined transmission from sources by conventional contact tracing.

Results. We analyzed 394 clusters with a total of 1285 patients. On the basis of molecular linkage only, 12.6\% of the secondary cases were attributable to transmission from a patient with smear-negative TB. The relative transmission rate among patients with smear-negative TB, compared with patients with smear-positive TB, was 0.24 (95\% confidence interval, 0.20-0.30). Secondary cases in clusters with an index patient with smear-negative TB more frequently had smear-negative status (odds ratio, 1.86; 95\% confidence interval, 1.18-2.93), compared with secondary cases in clusters with an index patient with smear-positive TB. Conventional contact tracing revealed that $26(6.2 \%)$ of the 417 sources, as identified by the Municipal Health Services, had smear-negative TB.

Conclusions. In The Netherlands, patients with smear-negative, culture-positive TB are responsible for $13 \%$ of TB transmission. Countries that have ample resources should expand their TB-control efforts to include prevention of transmission from patients with smear-negative, culture-positive pulmonary TB.

Mycobacterium tuberculosis is mainly transmitted by patients with pulmonary tuberculosis (TB). Microscopic examination of sputum smear specimens for the presence of M. tuberculosis, observed as acid-fast bacilli, is used worldwide to diagnose TB. Patients with sputum smear-negative TB are less infectious than patients with sputum smear-positive TB $[1,2]$. Nevertheless, patients with smear-negative, culture-positive pulmonary $\mathrm{TB}$

Received 16 April 2008; accepted 23 June 2008; electronically published 29 September 2008

Reprints or correspondence: Dr. Alma Tostmann, Dept. of Pulmonary Diseases (454), Radboud University Nijmegen Medical Centre, P0 Box 9101, 6500 HB, Nijmegen, The Netherlands (a.tostmann@ulc.umcn.nl).

Clinical Infectious Diseases 2008; 47:1135-42

(C) 2008 by the Infectious Diseases Society of America. All rights reserved. $1058-4838 / 2008 / 4709-0003 \$ 15.00$

DOI: $10.1086 / 591974$ are capable of transmitting M. tuberculosis [3, 4]. Light microscopy can detect mycobacteria at a minimum density of 5000-10,000 bacilli per $\mathrm{mL}$ of sputum, whereas the infectious amount is only a few organisms $[5,6]$. Therefore, persons in contact with patients with smear-negative TB are at risk of infection due to $M$. tuberculosis and the subsequent development of active TB $[2,7,8]$. However, there are limited quantitative data on the relative contribution of patients with smearnegative, culture-positive TB to TB transmission.

Recent studies in San Francisco [3] and Vancouver [4] that used molecular linkage estimated that, for $17 \%-20 \%$ of patients with TB, TB resulted from transmission by patients with smear-negative TB. However, these studies analyzed only 71 and 44 clusters, respectively.

In our study, we expanded on these observations by 
determining the contribution of patients with smear-negative, culture-positive TB to TB transmission in a large cohort in The Netherlands with use of both molecular linkage and data from contact investigations performed by the Municipal Health Services. The advantage of studying this topic among a large national TB cohort is the added confidence that may be gained by the large number of patients studied over a long period.

\section{METHODS}

Patients. Demographic and clinical information for all patients with TB diagnosed in The Netherlands during the period 1996-2004 was retrieved from The Netherlands Tuberculosis Register (NTR).

Molecular linkage. M. tuberculosis isolates from all patients with TB who had a positive culture result were subjected to standardized IS6110-based restriction fragment-length polymorphism typing to disclose linkage between patients with TB, as described elsewhere $[9,10]$. Isolates with $\leqslant 4$ IS6110 bands were subtyped using the polymorphic GC-rich sequence as a probe. Patients whose M. tuberculosis isolates had 100\% identical DNA fingerprints were clustered. In general, the first patient who received a diagnosis in a cluster was considered to be the index patient of that cluster.

The Beijing genotype was defined as described elsewhere [11]. The Haarlem genotype was defined according to IS6110 restriction fragment-length polymorphism and spoligotype patterns [12] and sequencing of the ogt gene [13].

Cluster selection. Data from the NTR were matched with data from the national M. tuberculosis DNA fingerprint database on the basis of sex, date of birth, date of TB diagnosis, and postal code, as described elsewhere [14]. Patients with a unique M. tuberculosis DNA fingerprint and clusters in which the index patient had only extrapulmonary TB were excluded from the analysis. Because sputum smear status has only been recorded in the NTR since 1996, we included only clusters from during or after 1996. To increase the chance that only new clusters were included, we excluded all clusters including cases from the period 1993-1995. No ethical approval was needed, because we used retrospectively gathered anonymous data.

Proportion of transmission caused by patients with smearnegative status. Our primary epidemiological outcome was the proportion of $\mathrm{TB}$ transmission caused by patients with smear-negative status. The transmission events associated with patients with smear-negative status were only calculated for clusters in which the smear status of the index patient was known. Secondary cases that were preceded only by cases of smear-negative TB were attributed to smear-negative transmission (i.e., transmission from a patient with smear-negative TB). All cases that occurred after any case of smear-positive TB were attributed to smear-positive transmission (i.e., transmission from a patient with smear-positive TB). For clusters in which the index patient's smear status was unknown, we used the 2 following extreme approaches as a sensitivity analysis: we determined the proportion of smear-negative transmission events assuming that all unknown smear results were positive, and we determined the proportion of smear-negative transmission events assuming that all unknown smear results were negative.

Relative transmission rate. Our secondary outcome was the relative rate of transmission by patients with smear-negative status. This rate indicates the proportion of transmission that is caused by patients with smear-negative $\mathrm{TB}$, compared with the proportion caused by patients with smear-positive TB. The relative transmission rate was calculated as the number of smear-negative transmission events per the total number of patients with smear-negative TB divided by the number of smear-positive transmission events per the total number of patients with smear-positive TB. The relative transmission rate was calculated by using all patients from the matched database, with exclusion of only those patients with extrapulmonary $\mathrm{TB}$, because they did not have a smear result recorded in the NTR.

Sensitivity analysis. We corrected our primary outcome, the proportion of smear-negative transmission events, for 2 possible biases. The reversed-order-of-diagnosis bias would most likely occur in clusters in which the first 2 patients had cases diagnosed within a few months of each other [3]. Therefore, we sequentially removed all clusters in which the first 2 cases were diagnosed within 1, 2, 3, 4, 5, or 6 months of each other and recalculated the proportion of smear-negative transmission events.

When the first 2 cases in a cluster are diagnosed $>2$ years apart, it is unlikely that the second case would result from recent transmission by the patient with the first case [15]. To determine the effect of this no-recent-transmission bias, we sequentially removed all clusters in which the first 2 cases were diagnosed $>2,3,4$, and 5 years apart and recalculated the proportion of smear-negative transmission events.

Epidemiological linkage. Clustered patients with TB are systematically reported to the Municipal Health Services. TB public health nurses contact these patients to investigate possible epidemiological links with previous patients in that same DNA fingerprint cluster with use of standardized questionnaires [16]. If an epidemiological link is found between 2 clustered patients with $\mathrm{TB}$, the index patient is registered as a source, and the patient with the secondary case is registered as a contact. A source is defined as a definite source if the source and the contact know each other's name, and the source is defined as a probable source if the source and the contact do not know each other's name but share an identical risk factor (e.g., location visited or risk group) [16]. We calculated the proportion of patients with smear-negative TB among all sources, as defined by the Municipal Health Service. 
Statistical analysis. To study possible risk factors for being in a cluster with an index patient with smear-negative TB, we used logistic regression to calculate ORs for the categorical variables, and depending on the distribution, we used either Student's $t$ test or the Mann-Whitney $U$ test for numerical variables. A threshold of $P<.05$ was used to define statistical significance. All analyses were performed with use of SPSS for Windows, version 14.0 (SPSS).

\section{RESULTS}

Patients. The NTR contained 13,064 patients with all forms of TB from January 1996 through December 2004; 9139 (70.0\%) of these patients had culture-positive TB. The National Institute for Public Health and the Environment determined the DNA fingerprint of $9347 \mathrm{M}$. tuberculosis isolates during this period. We matched the NTR database to the DNA fingerprint database, which resulted in a matched database of 7438 patients with TB. Because DNA fingerprinting can only be performed for patients with culture-positive TB, 7438 (81.4\%) of 9139 patients with culture-confirmed TB were matched. Exclusion of all patients with a unique DNA fingerprint, who were in a cluster that started before 1996, or who were in a cluster in which the index patient had only extrapulmonary TB resulted in a database of 394 clusters with a total of 1285 patients (figure 1).

Molecular linkage. Compared with index patients with smear-positive TB, index patients with smear-negative TB more frequently had pulmonary and extrapulmonary TB (OR, 2.58; 95\% CI, 1.40-4.78) and were less frequently born in The Netherlands (OR, 0.52; 95\% CI, 0.28-0.95) (table 1). Secondary cases in clusters with an index patient with smear-negative TB were more likely to have smear-negative TB (OR, 1.86; 95\% $\mathrm{CI}, 1.18-2.93)$ and were more frequently $>55$ years of age (OR, 1.87; 95\% CI, 1.16-3.00), compared with secondary cases in clusters with an index patient with smear-positive TB (table 2).

The median time from diagnosis of the first case to diagnosis of the second case was significantly shorter in clusters with an index patient with smear-positive TB than in clusters with an index patient with smear-negative TB (533 vs. 216 days, respectively; $P<.001$ ) (table 3 ). The smear status of the index patient was not associated with characteristics of the $M$. tuberculosis genotype family, because the proportion of Haarlem or Beijing strain did not differ between clusters with an index patient with smear-positive TB and clusters with an index patient with smear-negative TB.

Proportion of smear-negative transmission events. In total, 359 (91.1\%) of the 394 clusters had an index patient with a known smear result, and these index patients lead to 844 secondary cases (table 4). Ninety-two clusters had an index patient with smear-negative status, and 106 (70.7\%) of the 150 secondary cases were attributable to transmission from a pa-

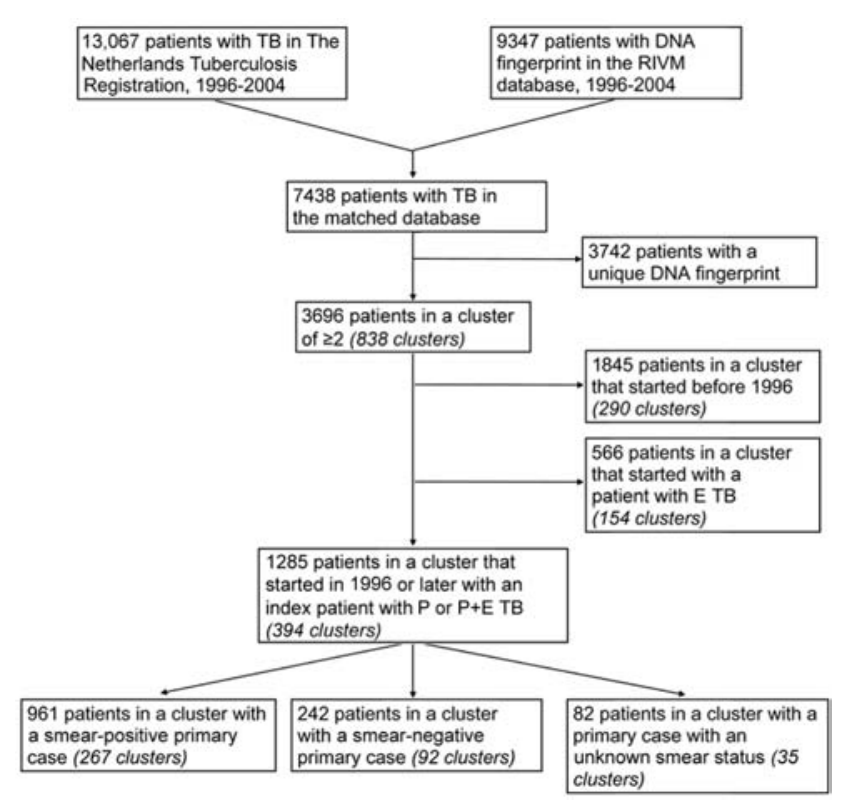

Figure 1. Flow chart of patient and cluster selection. E, extrapulmonary; P, pulmonary; RIVM, Mycobacteria Reference Laboratory at the National Institute for Public Health and the Environment (Bilthoven, The Netherlands); TB, tuberculosis.

tients with smear-negative status. The proportion of smearnegative transmission events was $12.6 \%$ (106 of 844 secondary cases resulted smear-negative transmission).

If all unknown smear results were assumed to be negative, $171(19.2 \%)$ of 891 transmission events would have been smear negative. Alternatively, if all unknown smear results were assumed to be positive, no extra smear-negative transmission events would have occurred (i.e., 106 [11.9\%] of the total 891 transmission events would have been smear negative) (table 4).

Sensitivity analysis. After correction for possible reversedorder-of-diagnosis bias, the proportion of smear-negative transmission events increased from $12.1 \%$ to $18.0 \%$. After correction for possible no-recent-transmission bias, the proportion of smear-negative transmission events decreased from $12.3 \%$ to $8.7 \%$.

Relative transmission rate. The relative transmission rate was calculated using all 7438 patients from the matched database. Of these patients, 3890 (52.3\%) had pulmonary TB, $2530(34.0 \%)$ had extrapulmonary TB, and 791 (10.6\%) had pulmonary and extrapulmonary TB; data were missing for 227 patients $(3.0 \%)$.

Overall, 1614 patients had smear-negative TB, and 2734 patients had smear-positive TB. Dividing the 106 smear-negative transmission events that occurred in the 1614 patients with smear-negative TB by the 739 smear-positive transmission events that occurred in the 2734 patients with smear-positive TB resulted in a relative transmission rate of 0.24 (95\% CI, $0.20-0.30)$. This means that patients with smear-negative pul- 
Table 1. Characteristics of the index patients.

\begin{tabular}{|c|c|c|c|c|c|}
\hline \multirow[b]{2}{*}{ Characteristic } & \multicolumn{4}{|c|}{ No. (\%) of index patients, by smear status } & \multirow[b]{2}{*}{ OR $(95 \% \mathrm{Cl})^{\mathrm{a}}$} \\
\hline & $\begin{array}{l}\text { Positive } \\
(n=267)\end{array}$ & $\begin{array}{l}\text { Negative } \\
(n=92)\end{array}$ & $\begin{array}{l}\text { Unknown } \\
(n=35)\end{array}$ & $\begin{array}{c}\text { All } \\
(n=394)\end{array}$ & \\
\hline \multicolumn{6}{|l|}{ Age, years } \\
\hline $0-14$ & $4(1.5)$ & $1(1.1)$ & $2(5.7)$ & $7(1.8)$ & $0.82(0.09-7.49)$ \\
\hline $15-34$ & $157(58.8)$ & $48(52.2)$ & $17(48.6)$ & $222(56.3)$ & 1 \\
\hline $35-54$ & $60(22.5)$ & $23(25.0)$ & $4(11.4)$ & $87(22.1)$ & $1.25(0.70-2.24)$ \\
\hline$\geqslant 55$ & $46(17.2)$ & $20(21.7)$ & $12(34.3)$ & $78(19.8)$ & $1.42(0.77-2.63)$ \\
\hline \multicolumn{6}{|l|}{ HIV status } \\
\hline Positive & $16(6.0)$ & $8(8.7)$ & $1(2.9)$ & $25(6.3)$ & $1.49(0.61-3.63)$ \\
\hline Negative & $27(10.1)$ & $9(9.8)$ & $8(22.9)$ & $44(11.2)$ & $1.00(0.45-2.21)$ \\
\hline Unknown & $224(83.9)$ & $75(81.5)$ & $26(74.3)$ & $325(82.5)$ & 1 \\
\hline \multicolumn{6}{|l|}{ Tuberculosis site ${ }^{b}$} \\
\hline Pulmonary & 235/264 (89.0) & 69/91 (75.8) & $26(74.3)$ & $330 / 390(84.6)$ & 1 \\
\hline Pulmonary and extrapulmonary & $29 / 264(11.0)$ & 22/91 (24.2) & $9(25.7)$ & $60 / 390(15.4)$ & $2.58(1.40-4.78)$ \\
\hline \multicolumn{6}{|l|}{ Sex } \\
\hline Male & $168(62.9)$ & $51(55.4)$ & $26(74.3)$ & $245(62.2)$ & $0.73(0.45-1.19)$ \\
\hline Female & $99(37.1)$ & $41(44.6)$ & $9(25.7)$ & 149 (37.8) & 1 \\
\hline \multicolumn{6}{|l|}{ Born in The Netherlands } \\
\hline No & $187(70.0)$ & $75(81.5)$ & $20(57.1)$ & $282(71.6)$ & 1 \\
\hline Yes & 77 (28.8) & $16(17.4)$ & $15(42.9)$ & $108(27.4)$ & $0.52(0.28-0.95)$ \\
\hline Missing data & $3(1.1)$ & $1(1.1)$ & $0(0)$ & $4(1.0)$ & $0.83(0.09-8.12)$ \\
\hline
\end{tabular}

monary $\mathrm{TB}$ were only 0.24 times as likely to spread $\mathrm{TB}$ as patients with smear-positive pulmonary TB.

Epidemiological linkage. From 1996 through 2004, the TB services of the Municipal Health Services identified 517 source patients during investigation of contacts among clustered patients with TB. Four hundred seventeen of these patients matched with our final database. A source patient was defined as a definite source of a case in another patient if the 2 patients knew each other by name and as a probable source if they did not know each other by name but had been in the same location. Twenty (5.4\%) of the 369 definite sources and $6(12.5 \%)$ of the 48 probable sources had smear-negative TB. Overall, 26 (6.3\%) of the 417 sources had smear-negative TB.

\section{DISCUSSION}

Our study revealed that $12.6 \%$ of the TB transmission in The Netherlands is caused by patients with smear-negative pulmonary TB. In clusters with an index patient with smear-negative status, almost $71 \%$ of the secondary cases were attributable to smear-negative transmission.

The conclusions of this study provide strong evidence that TB can be transmitted from patients with smear-negative status, confirming and expanding previous observations from smaller, urban studies $[3,4]$. In this respect, 3 independent studies in different settings converge on the conclusion that patients with smear-negative TB lead to approximately one-quarter as many cases of $\mathrm{TB}$ as do patients with smear-positive $\mathrm{TB}$ and are responsible for $10 \%-20 \%$ of transmission. We consider this result to be important for both developed and developing countries.

An intriguing finding is that, compared with index patients with smear-positive TB, index patients with smear-negative TB were associated with a disproportionate number of secondary cases with smear-negative TB (table 2). The reasons for this are not clear, although one possible explanation could be coinfection with HIV, because HIV infection is accompanied by high rates of smear-negative TB [17]. Unfortunately, because HIV status was unknown for the vast majority of the patients in our cohort, we could not analyze this possible explanation. Furthermore, bacteriological factors may have caused some clusters to have more patients with smear-negative TB than other clusters. In particular, we expected the Beijing strain to be more common in clusters with an index patient with smearpositive $\mathrm{TB}$, because the Beijing genotype is associated with high virulence, relapse, and treatment failure [18]. The Beijing genotype was observed in $9.0 \%$ of the clusters with an index patient with smear-positive TB, compared with $4.3 \%$ of the clusters with an index patient with smear-negative TB, but this 
Table 2. Characteristics of the secondary cases.

\begin{tabular}{|c|c|c|c|c|c|}
\hline \multirow[b]{2}{*}{ Characteristic } & \multicolumn{4}{|c|}{$\begin{array}{l}\text { No. }(\%) \text { of secondary cases, } \\
\text { by smear status of the index patient }\end{array}$} & \multirow[b]{2}{*}{ OR $(95 \% \mathrm{Cl})^{\mathrm{a}}$} \\
\hline & $\begin{array}{l}\text { Positive } \\
(n=694)\end{array}$ & $\begin{array}{l}\text { Negative } \\
(n=150)\end{array}$ & $\begin{array}{l}\text { Unknown } \\
(n=47)\end{array}$ & $\begin{array}{c}\text { All } \\
(n=891)\end{array}$ & \\
\hline \multicolumn{6}{|l|}{ Smear status } \\
\hline Positive & 277 (39.9) & $44(29.3)$ & $14(29.8)$ & 335 (37.6) & 1 \\
\hline Negative & 159 (22.9) & 47 (31.3) & $12(25.5)$ & $218(24.5)$ & $1.86(1.18-2.93)$ \\
\hline Missing data & $258(37.2)$ & 59 (39.3) & $21(44.7)$ & 338 (37.9) & $1.44(0.94-2.20)$ \\
\hline \multicolumn{6}{|l|}{ Age, years } \\
\hline $0-14$ & $40(5.8)$ & $3(2.0)$ & $6(12.8)$ & $49(5.5)$ & $0.37(0.11-1.22)$ \\
\hline $15-34$ & 377 (54.3) & 77 (51.3) & $19(40.4)$ & $473(53.1)$ & 1 \\
\hline $35-54$ & $193(27.8)$ & $38(25.3)$ & $10(21.3)$ & $241(27.0)$ & $0.96(0.63-1.48)$ \\
\hline$\geqslant 55$ & $84(12.1)$ & $32(21.3)$ & $12(25.5)$ & $128(14.4)$ & $1.87(1.16-3.00)$ \\
\hline \multicolumn{6}{|l|}{ HIV status } \\
\hline Positive & $34(4.9)$ & $6(4.0)$ & $1(2.1)$ & $41(4.6)$ & $0.78(0.32-1.90)$ \\
\hline Negative & $74(10.7)$ & $12(8.0)$ & $7(14.9)$ & $93(10.4)$ & $0.72(0.38-1.36)$ \\
\hline Unknown & $586(84.4)$ & $132(88.0)$ & $39(83.0)$ & 757 (85.0) & 1 \\
\hline \multicolumn{6}{|l|}{ Tuberculosis site } \\
\hline Pulmonary & 390/635 (61.4) & 76/136 (55.9) & $17 / 46$ (37.0) & $483 / 817(59.1)$ & 1 \\
\hline Pulmonary and extrapulmonary & 67/635 (10.6) & 13/136 (9.6) & $10 / 46(21.7)$ & $90 / 817(11.0)$ & $1.00(0.52-1.89)$ \\
\hline Extrapulmonary & $178 / 635(28.0)$ & 47/136 (34.6) & $19 / 46(41.3)$ & 244/817 (29.9) & $1.36(0.90-2.03)$ \\
\hline \multicolumn{6}{|l|}{ Sex } \\
\hline Male & $462(66.6)$ & 92 (61.3) & $29(61.7)$ & $583(65.4)$ & $0.80(0.55-1.15)$ \\
\hline Female & $232(33.4)$ & $58(38.7)$ & $18(38.3)$ & $308(34.6)$ & 1 \\
\hline \multicolumn{6}{|l|}{ Born in The Netherlands } \\
\hline No & $414(59.6)$ & $99(66.0)$ & $22(46.8)$ & $535(60.0)$ & 1 \\
\hline Yes & $221(31.8)$ & $37(24.7)$ & $24(51.1)$ & $282(31.6)$ & $0.70(0.46-1.06)$ \\
\hline Missing data & $59(8.5)$ & $14(9.3)$ & $1(2.1)$ & $74(8.3)$ & $0.99(0.53-1.85)$ \\
\hline
\end{tabular}

difference was not statistically significant (OR, 0.46; 95\% CI, 0.16-1.36). The small number of clusters with a patient who was positive for the Beijing strain (32 of the 394 clusters) in our database imposed an unfortunate limitation on our analysis.

Secondary cases associated with an index patient with smearnegative TB were older than secondary cases associated with an index patient with smear-positive TB. It is possible that the pathogenesis of $\mathrm{TB}$ in older people may differ from that in young people. More index patients with smear-negative TB than index patients with smear-positive TB were born outside The Netherlands. Perhaps a more extended time between infection and disease manifestation plays a role in this. This finding may also be related to the fact that more patients with $\mathrm{TB}$ who are born abroad more frequently have smear-negative $\mathrm{TB}$, compared with patients with $\mathrm{TB}$ who are born in The Netherlands.

Hernandez-Garduno et al. [4] either included or excluded all patients with only extrapulmonary $\mathrm{TB}$ in their analyses.
Patients with extrapulmonary TB most likely do not transmit TB unless the pulmonary site is unnoticed. Therefore, we excluded only the index patients with extrapulmonary TB only and not the secondary cases with extrapulmonary TB.

When using the epidemiological linkage data, only $6.3 \%$ of patients with TB had TB caused by transmission from a patient with smear-negative TB; the results of our molecular analysis indicate that $12.6 \%$ of patients with $\mathrm{TB}$ contracted $\mathrm{TB}$ from a patient with smear-negative TB. This difference is likely to be attributable to epidemiological links failing to reveal all contacts (e.g., unknown casual contacts).

The relative transmissibility of TB from patients with smearnegative TB was 0.24 (95\% CI, 0.20-0.30), which confirms the relative transmission rate of 0.22 ( $95 \% \mathrm{CI}, 0.16-0.32)$ that was found in Behr et al. [3]. This rate has implications for countries with a low incidence of $\mathrm{TB}$, especially those countries where the patient's smear status determines the isolation measures and the extent of contact investigation. In The Netherlands, contact tracing is performed for all patients with $\mathrm{TB}$, except 


\begin{tabular}{|c|c|c|c|c|c|}
\hline \multirow[b]{2}{*}{ Variable } & \multicolumn{4}{|c|}{ Index patients, by smear status } & \multirow[b]{2}{*}{ OR $(95 \% \mathrm{Cl})^{\mathrm{a}}$} \\
\hline & $\begin{array}{l}\text { Positive } \\
(n=267)\end{array}$ & $\begin{array}{l}\text { Negative } \\
(n=92)\end{array}$ & $\begin{array}{l}\text { Unknown } \\
(n=35)\end{array}$ & $\begin{array}{c}\text { All } \\
(n=394)\end{array}$ & \\
\hline \multicolumn{6}{|l|}{ Cluster size } \\
\hline $2-4$ & 224 (83.9) & $86(93.5)$ & $35(100)$ & $345(87.6)$ & 1 \\
\hline $5-10$ & $32(12.0)$ & $6(6.5)$ & $0(0)$ & $38(9.6)$ & $0.49(0.20-1.20)$ \\
\hline $11-20$ & $6(2.2)$ & $0(0)$ & $0(0)$ & $6(1.5)$ & \\
\hline $21-50$ & $5(1.9)$ & $0(0)$ & $0(0)$ & $5(1.3)$ & $\ldots$ \\
\hline $\begin{array}{l}\text { Time between diagnosis of the first case to diagnosis } \\
\text { of the second case, median days (IQR) }\end{array}$ & $216(69-672)$ & $533(221-1201)$ & $410(74-957)$ & & \\
\hline \multicolumn{6}{|l|}{ Beijing genotype } \\
\hline Yes & $24(9.0)$ & $4(4.3)$ & $4(11.4)$ & $32(8.1)$ & $0.46(0.16-1.36)$ \\
\hline No & $243(91.0)$ & $88(95.7)$ & $31(88.6)$ & 362 (91.9) & 1 \\
\hline \multicolumn{6}{|l|}{ Haarlem genotype } \\
\hline Yes & $50(18.7)$ & $16(17.4)$ & $6(17.1)$ & 72 (18.3) & $0.91(0.49-1.70)$ \\
\hline No & 217 (81.3) & 76 (82.6) & 29 (82.9) & $322(81.7)$ & 1 \\
\hline
\end{tabular}

NOTE. Data are no. (\%) of patients, unless otherwise indicated. IQR, interquartile range.

a The ORs were calculated for patients in clusters with an index patient with smear-negative tuberculosis versus patients in clusters with an index patient with smear-positive tuberculosis, using univariate logistic regression. Clusters in which the smear status of the index patient was unknown were not considered in these analyses.

b $P<.001$.

for patients with extrapulmonary TB for whom transmission can be practically excluded (e.g., vertebral TB). In The Netherlands, contact tracing is performed for both patients with smear-negative TB and patients with smear-positive TB; however, this is not the case in many other countries with a low incidence of TB.

Our study revealed that this contact-tracing policy is justified. Not only do the workers at a TB service search for patients with $\mathrm{TB}$ (i.e., contact tracing), they also search for the possible source patient. Contact tracing starts in the so-called first ring, which includes the closest contacts to the patients with TB, and is then expanded to the next ring, according to the yield of the ring [19]. The infectiousness of patients with smearnegative $\mathrm{TB}$ is especially important in light of multidrug-resistant and extremely drug-resistant TB; therefore, it is highly advisable to perform contact tracing for all patients with these forms of drug-resistant TB, irrespective of their smear status.

Our study was based on several assumptions. The first assumption was that the first patient in a cluster is the index patient of that cluster. This may not always be true. For example, the real source patient of that cluster could have received a diagnosis before the start of the study period. To increase the chance that only new clusters were included, we excluded all clusters in which there were patients from the period 19931995. Furthermore, patients with an identical DNA fingerprint may not have necessarily infected each other. Some strains are common among specific ethnic groups in The Netherlands, and these may be circulating strains in some patients' countries of origin. Therefore, there could have been more clusters of patients with identical DNA fingerprints that were not the result of transmission between the patients in the cluster. Because this might have occurred to the same extent in clusters with either index patients with smear-positive TB or index patients with smear-negative TB, we assume that this would have only a limited effect on our data. Sensitivity analyses revealed that both reversed-order-of-diagnosis bias and no-recent-transmission bias may have occurred in our study to a limited extent; thus, the outcome could be slightly overestimated or underestimated.

Approximately $68 \%$ of all patients with TB in The Netherlands have culture-confirmed TB; DNA fingerprinting can be performed only for those patients. Comparison of the 2 databases resulted in a match for $7438(81.4 \%)$ of the 9139 patients with culture-positive TB. This was concordant with a similar matching procedure performed with use of these same databases, by which no differences in sex, age, and nationality were found between patients with culture-confirmed TB who matched and patients with culture-confirmed TB who did not match [14]. Nonmatches were probably largely attributable to registration errors and, to a lesser extent, to patients not being registered in 1 of the 2 databases.

In The Netherlands, $\sim 80 \%$ of the laboratories use fluorescence microscopy. Fluorescence microscopy is more sensitive than conventional microscopy and will therefore detect more cases of smear-positive TB [20]. It is possible that, among the $20 \%$ of patients in our study cohort who had their cases diagnosed with use of conventional microscopy, fewer patients would have been determined to have smear-negative TB if their 


\begin{tabular}{|c|c|c|c|c|}
\hline \multirow[b]{2}{*}{ Variable } & \multicolumn{4}{|c|}{$\begin{array}{l}\text { No. of secondary cases, by smear } \\
\text { status of the index patient }\end{array}$} \\
\hline & Positive & Negative & Unknown & All \\
\hline Total & 694 & 150 & 47 & 891 \\
\hline \multicolumn{5}{|c|}{ Clusters with an index patient with smear-positive or smear-negative TB only } \\
\hline Secondary case attributable to smear-positive transmission & 694 & 44 & 0 & 738 \\
\hline Secondary case attributable to smear-negative transmission & 0 & 106 & 0 & 106 \\
\hline All & 694 & 150 & 0 & 844 \\
\hline \multicolumn{5}{|c|}{ If all patients with unknown smear results were considered to have smear-positive TB } \\
\hline Secondary case attributable to smear-positive transmission & 694 & 44 & 47 & 738 \\
\hline Secondary case attributable to smear-negative transmission & 0 & 106 & 0 & 106 \\
\hline All & 694 & 150 & 47 & 891 \\
\hline \multicolumn{5}{|c|}{ If all patients with unknown smear results were considered to have smear-negative TB } \\
\hline Secondary case attributable to smear-positive transmission & 694 & 21 & 5 & 720 \\
\hline Secondary case attributable to smear-negative transmission & 0 & 129 & 42 & 171 \\
\hline All & 694 & 150 & 47 & 891 \\
\hline
\end{tabular}

NOTE. The total number of index patients was 394; 267 had positive smear status, 92 had negative smear status, and 35 had unknown smear status. The total number of patients was 1285; 961 had positive smear status, 242 had negative smear status, and 82 had unknown smear status. The proportion of smear-negative transmission events was calculated by dividing the number of smear-negative transmission events by the total number of transmission events, using the 3 different scenarios shown. The proportion of smear-negative transmission events was $12.6 \%$ (106 of 844 secondary cases were attributable to smear-negative transmission), the proportion of smear-negative transmission events was $11.9 \%$ (106 of 891 ), and the proportion of smear-negative transmission events was $19.2 \%$ (171 of 891). TB, tuberculosis.

cases were diagnosed with use of fluorescence microscopy. Because the microscopy technique used for diagnosis is not registered in the NTR, we could not determine whether this affected the percentage of transmission by patients with smear-negative TB.

In countries with a high incidence of TB, microscopic examination of sputum smear samples is often the only available diagnostic test for TB. As a result, patients with smear-negative TB do not receive a diagnosis in a timely manner; thus, disease may further develop, initiation of treatment may be delayed, and further TB transmission may occur [21]. Although it is not known whether HIV-TB-coinfected patients for whom the result of sputum smear is negative are as infectious as we found in our study [17], our confirmatory finding that patients with smear-negative TB can transmit TB has implications for countries where HIV infection is endemic. HIV infection and AIDS are accompanied by high rates of smear-negative TB. For example, 25\%-61\% of the HIV-infected patients with TB in subSaharan Africa have smear-negative pulmonary TB [22]. More emphasis should be placed on the development of better TB diagnosis and the improvement of culture facilities in countries with a high incidence of TB [23].

In conclusion, our study suggests that $12.6 \%$ of the TB transmission in The Netherlands is caused by patients with smearnegative pulmonary TB. Although sputum smear analysis reveals most of the patients with infectious $\mathrm{TB}$, patients with sputum smear-negative TB should not be considered to be noninfectious. Therefore, we recommend that, in countries with a low TB burden and sufficient public health resources, contact investigation should be expanded to include patients with smear-negative $\mathrm{TB}$, in addition to patients with smearpositive TB.

\section{Acknowledgments}

We thank all Municipal Health Services, for contributing data to The Netherlands Tuberculosis Register, and Arnout Mulder, for excellent technical assistance at the mycobacteria laboratory of the National Institute for Public Health and the Environment.

Financial support. Royal Netherlands Tuberculosis Association, The Hague (to A.T., M.M.S., and N.A.K.), Poverty Related Infection Oriented Research (to A.T.), and The Netherlands Organization for Health Research and Development (to S.V.K. and S.V.).

Potential conflicts of interest. All authors: no conflicts.

\section{References}

1. Shaw JB, Wynn-Williams N. Infectivity of pulmonary tuberculosis in relation to sputum status. Am Rev Tuberc 1954; 69:724-32.

2. Grzybowski S, Barnett GD, Styblo K. Contacts of cases of active pulmonary tuberculosis. Bull Int Union Tuberc 1975; 50:90-106.

3. Behr MA, Warren SA, Salamon H, et al. Transmission of Mycobacterium tuberculosis from patients smear-negative for acid-fast bacilli. Lancet 1999; 353:444-9.

4. Hernandez-Garduno E, Cook V, Kunimoto D, Elwood RK, Black WA, FitzGerald JM. Transmission of tuberculosis from smear negative patients: a molecular epidemiology study. Thorax 2004; 59:286-90.

5. Yeager H Jr, Lacy J, Smith LR, LeMaistre CA. Quantitative studies of mycobacterial populations in sputum and saliva. Am Rev Respir Dis 1967; 95:998-1004.

6. Hobby GL, Holman AP, Iseman MD, Jones JM. Enumeration of tubercle bacilli in sputum of patients with pulmonary tuberculosis. Antimicrob Agents Chemother 1973; 4:94-104.

7. van Geuns HA, Meijer J, Styblo K. Results of contact examination in Rotterdam, 1967-1969. Bull Int Union Tuberc 1975; 50:107-21.

8. Elwood RK, Cook VJ, Hernandez-Garduno E. Risk of tuberculosis in 
children from smear-negative source cases. Int J Tuberc Lung Dis 2005; 9:49-55.

9. van Embden JD, Cave MD, Crawford JT, et al. Strain identification of Mycobacterium tuberculosis by DNA fingerprinting: recommendations for a standardized methodology. J Clin Microbiol 1993;31:406-9.

10. van Soolingen D, de Haas PE, Hermans PW, Groenen PM, van Embden JD. Comparison of various repetitive DNA elements as genetic markers for strain differentiation and epidemiology of Mycobacterium tuberculosis. J Clin Microbiol 1993;31:1987-95.

11. Kremer K, Glynn JR, Lillebaek T, et al. Definition of the Beijing/W lineage of Mycobacterium tuberculosis on the basis of genetic markers. J Clin Microbiol 2004; 42:4040-9.

12. Kremer K, van Soolingen D, Frothingham R, et al. Comparison of methods based on different molecular epidemiological markers for typing of Mycobacterium tuberculosis complex strains: interlaboratory study of discriminatory power and reproducibility. J Clin Microbiol 1999; 37:2607-18.

13. Rad ME, Bifani P, Martin C, et al. Mutations in putative mutator genes of Mycobacterium tuberculosis strains of the W-Beijing family. Emerg Infect Dis 2003; 9:838-45.

14. Kik SV, Verver S, van Soolingen D, et al. Tuberculosis outbreaks predicted by characteristics of first patients in a DNA fingerprint cluster. Am J Respir Crit Care Med 2008; 178:96-104.

15. Ferebee SH. Controlled chemoprophylaxis trials in tuberculosis: a general review. Bibl Tuberc 1970;26:28-106.

16. Lambregts-van Weezenbeek CS, Sebek MM, van Gerven PJ, et al. Tu- berculosis contact investigation and DNA fingerprint surveillance in The Netherlands: 6 years' experience with nation-wide cluster feedback and cluster monitoring. Int J Tuberc Lung Dis 2003; 7:S463-70.

17. Perkins MD, Cunningham J. Facing the crisis: improving the diagnosis of tuberculosis in the HIV era. J Infect Dis 2007; 196(Suppl 1):S15-27.

18. Abebe F, Bjune G. The emergence of Beijing family genotypes of $M y$ cobacterium tuberculosis and low-level protection by bacille CalmetteGuérin (BCG) vaccines: is there a link? Clin Exp Immunol 2006; 145 : 389-97.

19. Veen J. Microepidemics of tuberculosis: the stone-in-the-pond principle. Tuber Lung Dis 1992; 73:73-6.

20. Kivihya-Ndugga LE, van Cleeff MR, Githui WA, et al. A comprehensive comparison of Ziehl-Neelsen and fluorescence microscopy for the diagnosis of tuberculosis in a resource-poor urban setting. Int J Tuberc Lung Dis 2003; 7:1163-71.

21. Siddiqi K, Lambert ML, Walley J. Clinical diagnosis of smear-negative pulmonary tuberculosis in low-income countries: the current evidence. Lancet Infect Dis 2003; 3:288-96.

22. Storla DG, Yimer S, Bjune GA. A systematic review of delay in the diagnosis and treatment of tuberculosis. BMC Public Health 2008; 8: 15.

23. Getahun H, Harrington M, O'Brien R, Nunn P. Diagnosis of smearnegative pulmonary tuberculosis in people with HIV infection or AIDS in resource-constrained settings: informing urgent policy changes. Lancet $2007 ; 369: 2042-9$. 